\title{
Targeting cancer cell metabolism in glioblastoma
}

Current treatment for glioblastoma, which is dominated by radiotherapy and chemotherapy, is limited by toxicity to normal cells, resistance to therapy and high rates of recurrence. Now, writing in Nature, Parada and colleagues have identified a novel small-molecule inhibitor of oxidative phosphorylation, which exerts cancer-cell-specific toxicity and inhibits glioblastoma growth in mouse models.

Previous cell-based anticancer drug screens have predominantly identified agents that interfere with mitosis, replication or DNA damage and therefore target both healthy and cancer cells. Shi et al. therefore set out to identify candidate antitumour molecules that would not display toxicity towards normal cycling cells.

To do this, the authors used primary early-passage tumour cells, serum-free conditions and low oxygen tension to screen for potential anticancer compounds. In addition, to exclude nonspecific or anti-mitotic toxicity, the authors performed a counter screen against primary low-passage mouse embryonic fibroblasts (MEFs) and neonatal astrocytes.

The authors established primary 'high-throughput glioblastoma sphere' (HTS) cells by pooling sphere cultures from multiple tumours from a spontaneous glioblastoma mouse model with mutations in three glioblastoma-relevant tumour suppressors. A high-throughput chemical screen of 200,000 compounds in these HTS cells identified 61 compounds as specific inhibitors with halfmaximal inhibitory concentrations in the nanomolar range.

Further testing identified the benzimidazolinium Gboxin as a lead compound, which rapidly inhibited the growth of HTS cells, but not that of primary MEFs or astrocytes. Transcriptional microarray analysis and gene ontology studies in HTS cells revealed upregulation of expression of the transcription factor ATF4 and its stress response targets following treatment with Gboxin, as well as downregulation of survivin, which was followed by a molecular signature of apoptosis.

Mechanistically, Gboxin was found to accumulate inside glioblastoma cell mitochondria and disrupt primary glioblastoma cell metabolism.

First, the authors noted that Gboxin treatment suppressed expression of thioredoxin-interacting protein, a regulator of glucose uptake and consumption and a target of oxidative phosphorylation (OXPHOS). This lead to further in vitro studies that demonstrated Gboxin reduced the high mitochondrial membrane potential in HTS cells (which is characteristic of cancer cells and results in a high $\mathrm{pH}$ within the mitochondrial matrix), but not in MEFs. Gboxin also rapidly and irreversibly inhibited HTS cellular oxygen consumption rate with equal potency to that of well-characterized OXPHOS inhibitors.

Gboxin rapidly interacted with OXPHOS proteins, particularly complex $\mathrm{V}$, in a manner that was dependent on the proton gradient across the inner mitochondrial membrane of cancer cells.

Furthermore, structure-activity

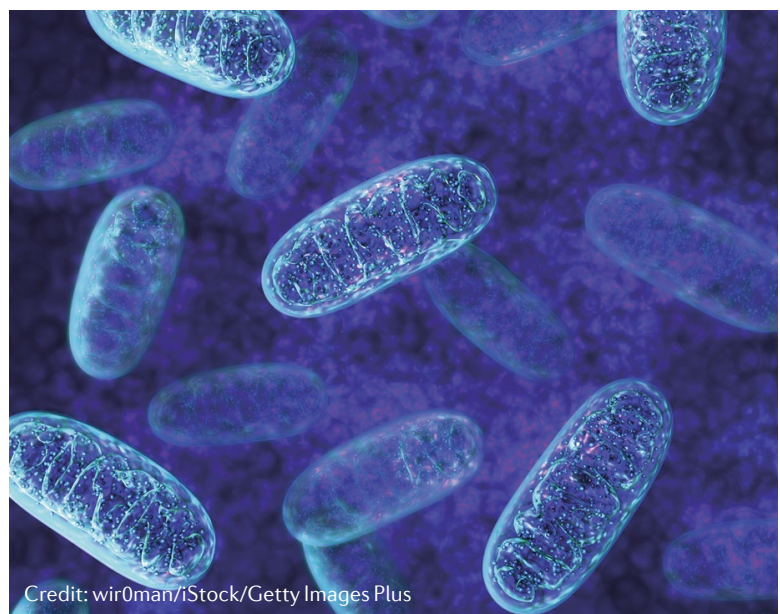

relationship studies determined that the positive charge on Gboxin is essential for its accumulation inside glioblastoma cell mitochondria.

A functional mitochondrial permeability transition pore (mPTP) - which can transiently open to expel excess reactive oxygen species and reduce membrane potential inside the mitochondrion - was found to be required for Gboxin resistance. Indeed, activity of the MPTP was blunted in Gboxin-sensitive HTS cells compared with resistant MEFs. Moreover, cyclosporin A-mediated blockade of the MPTP sensitized MEFs to Gboxin toxicity, leading to increased mitochondrial $\mathrm{pH}$, Gboxin accumulation, Gboxin association with OXPHOS proteins and cellular toxicity.

Notably, Gboxin toxicity extended to primary human glioblastoma cell cultures, in addition to other human cancer cell lines derived from diverse tumour types.

For in vivo studies, the authors identified a functional analogue S-Gboxin - that had excellent metabolic stability and enhanced plasma stability and pharmacokinetic properties. In mice, daily S-Gboxin injection following allograft flank implantation of HTS cells reduced tumour volume, cellular density and proliferation, and increased rates of survival. Furthermore, in patientderived xenograft models, in which glioblastoma cells were orthotopically implanted into the brains of mice, minipump-mediated delivery of S-Gboxin to the tumour area inhibited glioblastoma growth and increased survival without signs of toxicity.

In summary, this study has identified a novel small-molecule inhibitor of oxidative phosphorylation that exerts glioblastoma tumour-cell-specific toxicity, and highlights the potential of targeting the increased proton gradient and $\mathrm{pH}$ in cancer cell mitochondria as a general anticancer strategy.

Sarah Crunkhorn, Senior Editor, Nature Reviews Drug Discovery

This article is modified from the original in Nat. Rev. Drug Discov.

(https://doi.org/10.1038/d41573-019-00054-3).

ORIGINAL ARTICLE Shi, Y. et al. Gboxin is an oxidative phosphorylation inhibitor that targets glioblastoma. Nature 567, 341-346 (2019) 